\title{
Prospective Study in Carcinoma Larynx to Compare Two Regimens of Chemoradiation Consisting of Cisplatin with Conventional Radiotherapy and Cisplatin as Chemo Boost with Conventional Radiotherapy
}

\author{
Singh Yuvraj*, Prassad SN and Pramod \\ J.K. Cancer Institute, Kanpur \\ Submission: February 03, 2017; Published: February 22, 2017 \\ *Correspondence Address: Singh Yuvraj, J.K. Cancer Institute, Kanpur, Tel no: 9792928529; Email: dryuvrajbrd@gmail.com
}

\begin{abstract}
Introduction: A non-operative approach is favoured for patients in which surgery followed by radiation may lead to severe functional impairment particularly in a place where the bulk of carcinoma larynx patients are in advanced stages.

Aims and Objectives: To evaluate histopathologically proven advanced stage squamous cell carcinoma larynx patients and randomise into two arms to compare locoregional response, acute and chronic toxicities in the two arms.

Patients and Methods: Histologically proven non metastatic squamous cell carcinoma larynx stage 3 and 4 patients were registered and randomized into two arms. ARM I consisting concurrent chemo-radiotherapy of Inj. Cisplatin $100 \mathrm{mg} / \mathrm{m}^{2}$ three weekly with EBRT/ 60 Co OR LINAC/ 70 Gy / 35\# / 7 weeks / 2 parallel opposed fields. ARM II consisting of chemo boost as Inj Cisplatin $6 \mathrm{mg} / \mathrm{m}^{2}$ over last 15 fractions of treatment with EBRT / 60 Co OR LINAC / 70 Gy / 35\# / 7 weeks / 2 parallel opposed fields.

Results: Total 64 patients were registered, 32 in Arm I and 32 in Arm II. In Arm I, 10 pts.(31\%) showed complete response while in Arm II 11 pts.(38\%) showed complete response. Maximum number of patients developed Grade 1 and Grade 2 toxicities during 3rd and 4th weeks of RT in both the Arms with more number of treatment breaks in Arm II.

Conclusion: The study suggests that locoregional response is comparable in both the arms with more treatment related toxicities in Arm II resulting in more number of treatment breaks.
\end{abstract}

\section{Introduction}

The head and neck region contains the critical areas for, respiration, nutrition, one's expression, personal appearance. Treatments used in the management of laryngeal tumors can induce additional mutilations and cosmetic deformities, worsening the quality of life. A non-operative approach is favoured for patients in which surgery followed by radiation may lead to severe functional impairment particularly in a place where the bulk of carcinoma larynx patients are in advanced stages.

Combined modality treatment in the form of chemo-radiation is preferred for most advanced stage carcinoma larynx. It is a well known fact by now that tumor control could be enhanced by applying principles derived from radiotherapy fractionation to optimize the time factor in treatment scheduling. However, there are limitations to the radiotherapy dose escalation owing to the proximity of several critical normal structures in the head and neck area. This observation has led us investigate the promising schedules of radio sensitizing concurrent chemotherapy as an attempt to enhance local control in locally advanced carcinoma larynx.

\section{Aims and Objectives}

To evaluate histopathologically proven advanced stage squamous cell carcinoma larynx patients and randomise into two arms to compare locoregional response, acute and chronic 
toxicities in the two arms.

\section{Materials and Methodology}

A total of 64 patients of carcinoma larynx patients histologically proven Squamous cell carcinoma group stage 3 and stage 4 who were registered between Feb 2013 to Jan 2014 were studied using two different chemo radiotherapy schedules. Initially carcinoma larynx patient's histologically proven Squamous cell carcinoma group stage 3 and stage 4 were randomly placed into two groups.

\section{Group I}

This group comprised of randomly selected patients, having histopathologically proven Squamous cell carcinoma of larynx group stage 3 and stage 4 . All patients received concurrent chemo radiotherapy of

a. Inj. Cisplatin $100 \mathrm{mg} / \mathrm{m}^{2}$ three weekly with

b. EBRT/ 60 Co OR LINAC/ 70 Gy / 35\# / 7 weeks / 2 fields

\section{Group II}

This group will comprise of randomly selected patients, having histopathologically proven Squamous cell carcinoma of larynx group stage 3 and stage 4 . All patients received chemo boost as

a. Inj Cisplatin $6 \mathrm{mg} / \mathrm{m}^{2}$ on last 15 fractions of treatment with

b. $\quad$ EBRT / 60 Co OR LINAC / 70 Gy / 35\# / 7 weeks / 2 fields

\section{Radiotherapy technique}

a. Supine position

b. Patients underwent a pre-treatment simulation to work out the field borders which covered the primary tumor, disease extension and neck nodes

c. B/L fields were treated every day.

d. Phase II planning after 46 Gy.

\section{Assessment During Treatment}

From the commencement of treatment, all the patients included in the study were carefully and regularly assessed daily during treatment and weekly during planned gaps in treatment.

a. Detailed clinical evaluation for the tolerance of each patient to the delivered treatment was done by thorough local examination of the patient for local disease status along with observation of acute toxic side effects of radiation.

b. Radiation reactions were assessed by Radiation Therapy Oncology Group (RTOG) criteria and WHO toxicity criteria (Annexure-3) c. Radiation Therapy Oncology Group (RTOG) acute morbidity scoring criteria were relevant from day 1 , the commencement of radiation, through day 90 and thereafter, the RTOG criteria for late effects were utilized (Annexure-4).

d. Tumor response (both primary and nodal response) was assessed by WHO response criteria (Annexure-5).

\section{Assessment at the Completion of Treatment}

a. All the patients were assessed two weeks after the completion of treatment, to detect acute complications like mucositis, skin reaction.

b. Acute treatment related toxicity was graded using common terminology criteria for adverse events (RTOG) and late toxicity by RTOG criteria.

c. The tumor response was assessed by using the WHO criteria

d. Radiological assessment for tumor was done when indicated.

\section{Follow up}

All the patients were followed up regularly on OPD basis for a period of at least six months, weekly for four weeks in first month and then monthly.

a. At every visit, each patient was clinically evaluated for local control of disease and treatment related complications.

b. The patients were assessed for any evidence of distant metastasis during each follow up.

c. To evaluate the local disease control, local examination using inspection, palpation was done at each follow up and response was assessed.

d. On the suspicion of any local recurrence, biopsy was taken for histopathology and correlated clinically.

e. To evaluate the distant metastasis detailed history pertaining to any symptoms was taken and general physical examination of patients was done.

f. In case of suspicion, relevant investigations were done to rule out the presence of distant metastasis.

g. The QOL was assessed at the beginning of treatment, on the day of completion of treatment and one month after completion of planned treatment using University of Washington QOL questionnaire.

The results of the study regarding safety, tolerability, toxicity and response in all the groups were documented.

\section{Statistical Analysis}

The data thus obtained was assessed, analyzed and compared to find out difference in all the groups in terms of tumor response 
and quality of life. The chi square test of significance was used to determine whether the observed results are statistically significant or not. P value less than 0.05 is significant. $P$ value less than 0.01 is highly significant.

\section{Observations and Results}

a. Total 64 patients were enrolled, 32 in each arm.

b. MALE: FEMALE ratio is $31: 1$. MAXIMUM NO. OF PTS AT PRESENTATION WERE BETWEEN 51 - 60 yrs. age group. Majority of pts (31\%) had addiction habits of biddi/ cigarette smoking with duration more than 20 yrs. Majority of pts. (78.1\%) belonged to rural background.

c. The complete response in Arm I when compared to Arm II ( $31 \%$ Vs 38\%) was found to be not significant (P value $0.06)$

d. Maximum no. of pts developed gr1 \&gr 2 acute toxicities during $3 \mathrm{rd}$ and 4 th wks of $\mathrm{rt}$

Toxicities were higher in arm ii ( $p$ value significant .03)

e. Maximum no. of pts developed gr1 \& gr 2 dysphasia during 3rd and 4 th wks of RT.
Toxicities were comparable in both the arms.

f. Arm I: $22 \%$ and $22 \%$ of pts (maximum no.) developed gr1 \& gr2 haematological toxicities respectively during $3^{\text {rd }}$ and 4 th wks of rt.

g. Arm II: $34 \%$ and $40 \%$ of pts (maximum no.) developed gr1 \& gr2 haematological toxicities respectively during $3^{\text {rd }}$ and 4 th wks of rt.

h. toxicities were higher in arm II ( $p$ value significant .04)

i. No intervention (Ryles tube/ Tracheostomy) was required in 59\% of pts. In arm I and $40 \%$ in arm II.

j. $\quad$ No treatment interruption was required in $72 \%$ of pts. in arm I and 62\% in arm II

\section{Conclusion}

The study suggests that locoregional response is comparable in both the arms with more treatment related toxicities in Arm II resulting in more number of treatment breaks.

\section{Acknowledgement}

It is my privilege to acknowledge my sincere gratitude to my wife Dr Jigyasa for her guidance and support.

\section{Your next submission with Juniper Publishers will reach you the below assets}

- Quality Editorial service

- Swift Peer Review

- Reprints availability

- E-prints Service

- Manuscript Podcast for convenient understanding

- Global attainment for your research

- Manuscript accessibility in different formats

( Pdf, E-pub, Full Text, Audio)

- Unceasing customer service

Track the below URL for one-step submission https://juniperpublishers.com/online-submission.php 